2000 mittelst Koli vollig in baldriananures Kali tmwandalt; es wer nun noch darzuthun, ob es fahiz wäre, vich durch oxydirenden Binflafs der Luft obenfalls in Baldriandãure gu rerwandeln. Man weifs, dafe Alkohol and Holzgeist, mittelst Platinsehwarz und Luft leicht, ersterer in Esuigazure, letzterer in Ameisenslure übergehen; ich habe mich nun überzeugt, dafs dan Kartoffelfunetäl wich gerade on vorhält. Man muls indeasen hierzu das Platiaschwarz vorber erhitsen und dan Oel nur tropfenweise darauf giefsen. Stellt man dtesen Versuch in einer oben offenen, in einet Schale mit Wasser stelienden Glocke an, so bemerkı men, dafo an ihren Wanden eine Flissigkeil hinabrinat, weiche dem Wasser eine saure Resction ertheill. Durch Sättigen des letateren mit Barytwaseer, Verdampfen und Zorsetzen des Rückstandes mit Phosphorsiore, orhäit maul tiue Flüxsigkeit, welche alle Eigenschaften der Baldriansäure besitat.

(Aunal. de Chim. el de Phys. T. LXXV. p. 193.;

\title{
Ueber die wachsartige Materie des Zuckerrohrs;
}

von Acequin. Apotheker in Neu-Orleam.

Sehr riele Phanzen schwitzen anf ihren Blātern oder Frtichten eime Substanz aus, welcher man den Namen regetabilisehes Wachs gegeben hat. Der Chemiker ' $T$ inges in Fonf erwahnte sie zuerat, apiter fand oie Prougt noeh suf vioten Frächten, den Pfaumen und Kirschen, auf fast allen Brttera, namentlich den graugränen. Die Kobs- und lrigarten, mehrere Gramineen, die Rosen liefern es ebenfalls. Die in den Tropenländern geammelten Flascheakürbisse sind 
mit oiner dicken Lage dieser Mlaterie bedeckt; keino Panaze enthät aber so viel daron als das Zuckerrohr.

Nicht alle Palenzen liefern dieselbe Waterie; es ist sogar wahrscheinlich, dafs sie immez in thren physikaliochen and chemischen Eigensebaften etras rerschieden lst und dafs jede Pfanze eine thr elgeathümliche Wachsart producirt. Die von dem Zuckerrohr gewonnene Subatanz hat einize Analogie mit dem Myricawachs und wurdo, so viel mir bekannt iut, niemals anteruncht. Ich habe es bei der vergleichenden Analyse des geatreiften and des Otoheitischen Zuckerrohm erwihnt, ohne aber seine Gigenschaften weiter 20 untersuchen, weil ich mir damals nicbt genug darou verechuffen konnte. Ich nenne diese Subutauz Cerosin, (von xegos, Wachs) was an seine Zusammensetzung and einige seiner wichtigaten Eigenschaften erinuern soll.

Diese Substanz findet sich am reiehlichsten aof der Oburfläche der Rinde des violetten, wie auf allen anderen Varietäten dea Zuckerrohrs. Die stengelumfasmende Basis der blätter ist ebenfalla daron bedeckt. Es bildet einen weifsen oder graugrünen, an der linde adbärirendeu Staub, der mittelat eines Measery leicht abgeschabt werden kano. Dus gestreifte Zuclerrohr liefert ebenfalli viel davon, das otaheitische ser kaum $1 / 9$ and das creolische last nichts. Auf letzterem bildet es nur oinen dïnnen Ring on der Basis eines jeden Knotens. Das schlechteste Zuckerrohr liefert an meloten davon; das violette ist sehr hart, holzig ond enthält werig. Saft ${ }^{*}$ ). Um es rein zu erhalten, wurde es in kaltem Alkohol von $35-33^{\circ}$ macerirt, wodurch eine violette, dem damit gemengten Chlorophyll äholiche Materie

-) Gestrelftes Zockerrohr in Batavia und Jars.

Otabeitiecbes " in Otabeitl.

Creollsches " ill Mralabar, Bengalen, Bourbon. 
entrogen warde; nech wehrwaliger Behendlung warde ec dann in kocbondem Alkohol von $33^{\circ}$ apfgelöat und nach der Enternang det Altoholo im Waserbade geachmolzen.

Dio Materie ist dann unjöslich in Waseer, fant unlöalich In kaltem Alkohol, röllig löalich aber in kochendern, workas ex vich beim Erkalten nicht abetzl. En gesteht dagegen sa eiber opalixirenden, eiber weingeiotigen Seifenlösung ahnlichen Mase. 4 Gren devon raichen hin, om 1 Unse Alkohol, dem Opodeldock glejch, geatehen su machen. Sie ist valöalich in kaltem Aether, schwer löalich in warwem und setat sich darau belm Erkalten in kleinen körnigen Kryatallea ab.

Sio ist gelblich, wehr hart, leicht an einem weifuen Pulver werroiblich und brennt, in Form einer Kerse, wie Wachs oder Wallsuth. Sie schmilat bei $82^{\circ} \mathrm{C}$. nnd wird bei $80^{\circ}$ wieder fest. Das opec. Gewicht jst 0,061 bei $10^{\circ}$. Sie ist geruchlos, verbindet wich sehr schwer mit Alkalien und vor indert sich nicht an der Luft.

Um we in krystelliniocher Forna en erhalten, schmilzt man sie im Waserbade nnd lifnt langsam erkalten. Wenn die Obertiliche eratarrt ist, lafat man durch eine eingenchmnt. sene Oeffnung den noch flüasigen Theil auefliefsen, no das Innere aludann eine Menge echr deutlicher, abgentumpfter Nedela soigt. Man mufo su diesem Vorsushe indeseen wenigaten ein Pfond der Materie anwenden.

Dieac Subotans iat identisch ouf allen Varietäten des Zurkerrohrs. 153 Stengel dea violetten Zuckerrohrs licferten durch Abschaben $170 \mathrm{Grm}$. daron; es lat indeseen anzunebmen, dafo anf diesem Wege nur die Finlfie der wahren Mienge erhalten worde.

Beiro Dnrchpressen des Zuckerrohm durch dio Mühle, um dea Saft su gewionen, lôat sich ein Theil dieser Ma- 
Dumas, aber dis Zusanamenestreng des Corosin so 173

terie dareh den Druck los uad sehwimme dann anf dem Safto ale weifoes Pulrer.

Erbitzt man dena don Saft zam Sieden, ohno Ralkmilch suxuetsen, so Irfat sich der Schum corgfillif sammoln; nach dem Wachen und Maceriren mit schwachem Alkohol wird er getrocknet und wiederholt mit kochendem Alkohol bohandelt; man erhält af diese Art eine gröfuero Aubbeute ron Corosin, welcher Indenen immer etwa grta gefírbt iat.

Beim Pressen des Zuckerrohre lönt iich nicht alles Corosin von den Stengeln los; 30 Litre Saft deo gestreiften Rohrs gaben 22,5 Grm. grünes Cerosiu. Bei elnerm anderen Veranch erhielt ich durch Abschaben ron einem violetten Rohr mehr als 2 Grm.

Ein Zuckerrohrfeld liefert fant 18000 Stengel; folglich würden diese 36 Kilogr. Cerosin geben. Eine Pflanzung, die jahrlich 300 Morgen subsat; könnte siso mohr als 10000 Rilogr. Ceronin liefern.

(Avn. de Chim. et de Phỵr. T. LXXV. p. 218)

\section{Ueber die Zusammensetzing des Cerosin's; ron J. Dumas.}

Ich habe der vortthenden Beachreibang nichte hinessofügen, sie ist vollkowmen gensn. En itt heopteichlich die Zusummensetzung dee Ceronin's welche wich beschartigt hat. Um sie aussumittein, lösto tch die Materio in kochendew Alkohol, liefs sie durch Drkalten krystallisiren und trackacic die mit Wawer gewaschenen Erystalle. 